\title{
A new coherent paired state with possible applications to fluctuation-dissipation phenomena
}

\author{
R F Bishop and A Vourdas $\dagger$ \\ Theoretical Physics Group, Department of Mathematics, University of Manchester Institute \\ of Science and Technology, PO Box 88, Manchester M60 1QD, UK
}

Received 1 July 1986, in final form 6 February 1987

\begin{abstract}
We extend our previous work on coherent paired states associated with the Lie group SU(1, 1). Whereas the earlier states were defined with respect to a single type of canonical boson or (linear) quantum harmonic oscillator, the new states are defined in terms of two distinct types of bosons or oscillators. The new coherent states may again, on the one hand, be viewed as ordinary (Glauber) coherent states in the two-boson Hilbert space spanned by arbitrary numbers of two distinct Bogoliubov quasiparticles associated with the original bosons via a generalised Bogloiubov transformation. Alternatively, expressed wholly in terms of the original bosons these new coherent states are reached from the ordinary coherent states via a unitary (pairing) transformation which is shown to be associated with the entire so-called discrete series of representations of the group $\operatorname{SU}(1,1)$. As an important illustration of the use of these states and transformations, we study in detail a rather general class of quantum Lagrangians which includes the damped linear harmonic oscillator. We thereby illustrate their possible usefulness in applications to quantum many-body or field-theoretic processes involving fluctuation-dissipation phenomena in general.
\end{abstract}

\section{Introduction}

The existence of self-bound clusters of particles within a many-body system of like particles plays a fundamental role in condensed matter theory. As is well known, such clusters are intimately related to the existence of a lower (non-perturbative) vacuum state for the system and hence to the possibility of a new macroscopic phase. Cooper pairs and the associated superconducting phase provide a typical example.

In a recent paper (Bishop and Vourdas 1986, hereafter referred to as I) we considered the problem of pairing within a many-body system of identical bosons. In terms of a set of boson creation and annihilation operators $a^{\dagger}$ and $a$, respectively, we made initial connection with the idea of correlated pairs by focusing attention on the quadratic operators $a^{2}, a^{\dagger 2}$ and $a^{\dagger} a$. In particular we noted how these operators could be arranged so as to close under commutation into the $\mathrm{SU}(1,1)$ algebra. We then also considered a very particular unitary representation of the corresponding Lie group which is realised with the operators $U_{2}=\exp \left(z a^{+2}-z^{*} a^{2}\right) \exp \left(i \lambda a^{\dagger} a\right)$. In this way we were led very naturally to the Bogoliubov canonical transformation, $U_{2} a U_{2}^{\dagger}=\mu a+\nu a^{\dagger} \equiv b$. The new operators $b$ and $b^{+}$respectively annihilate and create the Bogoliubov quasiparticles. The presumption is that in the phase where pairing physically occurs, the so-called

$\uparrow$ Present address: Fachbereich Physik, Philipps-Universität Marburg, Mainzergasse 33, D-3550 Marburg, West Germany. 
perturbative vacuum $|0\rangle$, which is the vacuum for a-type bosons $(a|0\rangle=0)$ is not the real one. Instead there exists a lower vacuum, $|0\rangle_{b}$, which corresponds to that for b-type bosons. This is defined by the relation $b|0\rangle_{\mathrm{b}}=0$, or equivalently as $|0\rangle_{\mathrm{b}}=U_{2}|0\rangle$.

Now, although the algebra $\mathrm{SU}(1,1)$ arose as outlined above from a general discussion of pairing, it is important to realise that the pairing operators do form only a special representation of the algebra. In other words, the Casimir operator for the algebra takes only a particular value. It is natural, therefore, now to proceed by exploring other representations of the $\mathrm{SU}(1,1)$ group, and in this paper we accordingly investigate the so-called discrete series of representations. We show that, whereas our previous representation could be associated with quantum states which could be realised by the ordinary harmonic oscillator (for which the operators $a$ and $a^{\dagger}$ may be thought of as the usual ladder operators), this is no longer the case for the discrete series of representations. Instead, we show how they may very profitably be associated with the damped harmonic oscillator, which may in turn itself be described with the help of not one but two commuting bosonic operators $a_{1}$ and $a_{2}$. We show how to construct for this case the analogue $V_{2}$ of the previous unitary operator $U_{2}$, and show how it correspondingly leads to a canonical transformation between the original operators $a_{1}$ and $a_{2}$ and two new operators $b_{1}$ and $b_{2}$. We refer to this transformation as a generalised Bogoliubov transformation.

Again, the operator $V_{2}$ is used to construct new coherent states, which turn out once more to be ordinary coherent states with respect to the transformed operators $b_{1}$ and $b_{2}$, but which have extremely interesting properties in connection with the original operators $a_{1}$ and $a_{2}$. Interestingly, these states have also very recently been introduced by Caves and Schumaker (1985) into quantum electronics as an extension of the older so-called squeezed states (Yuen 1976, Walls 1983). Within the context of quantum electronics, it is the properties of these states that are related with the uncertainty principle that are of most interest, and hence which have been most studied. By contrast, we study them here in connection with the generalised Bogoliubov transformation and, more widely, show how they may very profitably be employed in the context of quantum many-body theory. For example, we show how the generalised Bogoliubov transformation may be used to study Hamiltonians of the general form

$$
H=\omega_{1} a_{1}^{\dagger} a_{1}+\omega_{2} a_{2}^{\dagger} a_{2}+\kappa a_{1}^{\dagger} a_{2}^{\dagger}+\kappa * a_{1} a_{2}
$$

where $a_{1}$ and $a_{2}$ are two independent (commuting) boson destruction operators, which obey the usual canonical commutation relations. Just such Hamiltonians have been studied, for example, in the context of quantum optics by Mollow and Glauber (1967) as a model suitable for studying the phenomenon of parametric amplification. In this case the transformation maps the above Hamiltonian either into diagonal form for two free modes

$$
H_{1}=\varepsilon_{1} b_{1}^{\dagger} b_{1}+\varepsilon_{2} b_{2}^{\dagger} b_{2}
$$

or into the Hamiltonian of the single-mode damped harmonic oscillator,

$$
H_{2}=\varepsilon\left(b_{1}^{\dagger} b_{1}-b_{2}^{\dagger} b_{2}\right)+\mathrm{i} \Gamma\left(b_{1}^{\dagger} b_{2}^{\dagger}-b_{1} b_{2}\right)
$$

where in each case $b_{1}$ and $b_{2}$ are another pair of independent canonical boson operators. Whether $H$ is mapped onto $H_{1}$ or $H_{2}$ depends on whether the parameter $\Delta \equiv \frac{1}{4}\left(\omega_{1}+\omega_{2}\right)^{2}-|\kappa|^{2}$ is positive or negative respectively.

In the present paper we are more interested in the latter case of the damped harmonic oscillator, for which the Hamiltonian $\mathrm{H}_{2}$ has been previously studied in the literature (Feshbach and Tikochinsky 1977, Ghosh and Hasse 1981). Rather more 
generally, there are many physical phenomena which may be associated with models wherein an oscillator loses its energy within some many-body medium which also gives rise to it. In such descriptions the overall energy is, of course, conserved since just as some oscillators are damped, so others will be correspondingly amplified. A typical classical counterpart of the quantum systems that we have in mind is a system of many pendula coupled together by springs. Within quantum optics, these fluctuation-dissipation phenomena have mainly been studied via the Langevin equation approach (Senitzky 1960, 1961, Lax 1966a, b). The basic process is that the damping and amplification cause a splitting of each otherwise unperturbed energy level $(\Omega)$ into two neighbouring ones $(\Omega \pm \varepsilon)$. The mathematical consequence of this, namely that the associated representations that we now consider in this paper can only be studied with the use of two 'dual' bosonic operators, is then easily intuited.

In the present paper we explicitly construct the vacuum, other coherent states and, more generally, other states which are particularly appropriate for such problems. This is important, not only because of applications of the above example, but also because there are other problems related to it which may be of even greater significance. We have in mind here the many examples in quantum field theory and quantum many-body theory where double-well (or, more generally, multi-well) potentials play a key role. In these cases we have a flow of energy into and out of each well through quantum tunnelling. Again, the energy levels which each well would separately sustain in isolation now become split. Our hope is that the present work may be used to gain a further understanding of the vacuum and other states in such multi-well problems, and more generally in field theories with instantons (see, e.g., Coleman 1979). Where our work seems to be of particular promise here is that whereas these problems have in the past usually been studied with semiclassical (WKB) techniques, our own formalism is fully canonical.

The Feshbach-Tikochinsky Hamiltonian is based on a dual-coordinate formalism. It has been argued that, rather than describing the damped quantum harmonic oscillator, it should be viewed as a fictitious Lagrangian system with two degrees of freedom, the equations of motion for which are those of a damped oscillator and its corresponding anti-damped counterpart. Ghosh and Hasse (1981) in particular have criticised the model due to the lack of normalisability of the states. We go to some pains to address their comments in the present context. We stress that the model may still find real practical applicability in fluctuation-dissipation phenomena, in just the same way that resonant states (and their corresponding anti-resonant counterparts) have been used as very profitable approximations to many collective aspects of quantum many-body systems.

In $\S 2$ we introduce the new coherent states associated with the group $\mathrm{SU}(1,1)$ that we previously introduced in I. We also show explicitly how the generalised Bogoliubov transformation is generated, and we examine in some detail the properties of the associated new coherent states. After the discussion in $\S 3$ of the illustrative application of these results to the problem that we have already mentioned, we conclude in $\$ 4$ with some more detailed remarks about the formalism and with some comments on its further extension and other possible uses.

\section{Generalised Bogoliubov transformations and coherent states}

As in I, we let $K_{+}, K_{-}$, and $K_{0}$ be three operators which are closed under commutation 
and which satisfy the Lie algebra of $\operatorname{SU}(1,1)$, namely

$$
\left[K_{0}, K_{ \pm}\right]= \pm K_{ \pm} \quad\left[K_{-}, K_{+}\right]=2 K_{0} .
$$

The Casimir operator (which, by definition, commutes with each of these three operators) is defined as

$$
K^{2} \equiv K_{0}^{2}-\frac{1}{2}\left(K_{+} K_{-}+K_{-} K_{+}\right)
$$

By Schur's lemma this Casimir operator simply reduces for irreducible representations into a multiple of the unit operator $I$. Since the operators $K^{2}$ and $K_{0}$ commute, they can be simultaneously diagonalised, and we can thus define a suitable set of basis vectors $|k ; \mu\rangle$ by the relations

$$
\begin{aligned}
& K^{2}|k ; \mu\rangle=k(k-1)|k ; \mu\rangle \\
& K_{0}|k ; \mu\rangle=\mu|k ; \mu\rangle .
\end{aligned}
$$

Since $\operatorname{SU}(1,1)$ is a non-compact group, the quantum number $k$ is not constrained to take only discrete values. The various possibilities which form the spectrum of eigenvalues have been well studied elsewhere (Bargmann 1947, Barut and Fronsdal 1965, Holman and Biedenharn 1966, Barut and Girardello 1971, Perelomov 1977). The representations considered in I correspond to the eigenvalues $k=\frac{1}{4}, \frac{3}{4}$. By comparison, in this paper we consider the so-called discrete series of representations for which $k$ is positive and integral or half-integral, $k=\frac{1}{2}, 1, \frac{3}{2}, \ldots$ For a given representation $k$ in this series, the basis vectors are further specified by the quantum number $\mu \equiv k+m$, where $m$ is a non-negative integer, $m=0,1,2, \ldots$ It is not difficult to show that the action of the operators $K_{ \pm}$on this basis set of vectors is given as follows:

$$
K_{ \pm}|k ; \mu\rangle=[\mu(\mu \pm 1)-k(k-1)]^{1 / 2}|k ; \mu \pm 1\rangle \text {. }
$$

Before presenting our new results it is worthwhile briefly to recall our earlier unitary representation of the group $\mathrm{SU}(1,1)$ which was realised in I with the unitary operators

$$
\begin{aligned}
& U_{2}(\rho, \theta, \lambda) \equiv \exp \left(-\frac{1}{4} \rho \mathrm{e}^{-\mathrm{i} \theta} a^{\dagger 2}+\frac{1}{4} \rho \mathrm{e}^{\mathrm{i} \theta} a^{2}\right) \exp \left(\mathrm{i} \lambda a^{\dagger} a\right) \quad \rho, \theta, \lambda \in \mathbb{R} \\
& U_{2}^{\dagger} U_{2}=I=U_{2} U_{2}^{\dagger}
\end{aligned}
$$

where $a$ and $a^{\dagger}$ are standard annihilation and creation operators obeying the canonical boson commutation relations, $\left[a, a^{\dagger}\right]=I$. In this case the generators of the corresponding algebra are given by

$$
K_{+} \rightarrow \frac{1}{2} a^{+2} \quad K_{-} \rightarrow \frac{1}{2} a^{2} \quad K_{0} \rightarrow \frac{1}{2} a^{\dagger} a+\frac{1}{4}
$$

from which it is trivial to evaluate the Casimir operator of (2) as

$$
K^{2} \rightarrow-\frac{3}{16} I=k(k-1) I \quad k=\frac{1}{4}, \frac{3}{4} .
$$

It is now simple to see that in this case the basis vectors of (3) are just the states $\mid n$ ) of definite boson number

$$
a^{+} a|n\rangle=n|n\rangle \quad|n\rangle=(n !)^{-1 / 2}\left(a^{\dagger}\right)^{n}|0\rangle .
$$

Making use of (4) and (6)-(8), it is now easily seen that for even particle number, $n=2 N$, we have the $k=\frac{1}{4}$ representation, and the state $\langle 2 N\rangle$ is simply equivalent to the state $\left|\frac{1}{4} ; N+\frac{1}{4}\right\rangle$ in the notation of (3). In particular the vacuum state $|0\rangle$ containing no bosons is identical to the state $\left.\mid \frac{1}{4} ; \frac{1}{4}\right)$. Similarly the $k=\frac{3}{4}$ representation maps onto the states of odd particle number, $n=2 N+1$, and the state $|2 N+1\rangle$ in the notation 
of $(8)$ is equivalent to the state $\left|\frac{3}{4} ; N+\frac{3}{4}\right\rangle$ in the notation of $(3)$. The lowest state here is the single-boson state $|1\rangle \equiv\left|\frac{3}{4} ; \frac{3}{4}\right\rangle$. It is clear that the set of states

$$
|2 N\rangle \equiv\left|\frac{1}{4} ; N+\frac{1}{4}\right\rangle \quad|2 N+1\rangle \equiv\left|\frac{3}{4} ; N+\frac{3}{4}\right\rangle
$$

for $N=0,1,2, \ldots$, comprises an orthonormal basis which spans the entire Hilbert space $H_{1}$ of the harmonic oscillator.

After these introductory remarks we now consider the so-called discrete series of representations of $\mathrm{SU}(1,1)$, which clearly are associated with quantum states which cannot be realised by the single (undamped) oscillator above. As is already well known (Bargmann 1947, Barut and Fronsdal 1965, Holman and Biedenharn 1966, Barut and Girardello 1971, Perelomov 1977), these states may be expressed in terms of a pair of commuting bosonic operators $a_{1}$ and $a_{2}$

$$
K_{+} \rightarrow a_{1}^{+} a_{2}^{+} \quad K_{-} \rightarrow a_{1} a_{2} \quad K_{0} \rightarrow \frac{1}{2}\left(a_{1}^{+} a_{1}+a_{2}^{+} a_{2}+1\right)
$$

which satisfy the commutation relations of (1) when

$$
\begin{aligned}
& {\left[a_{1}, a_{1}^{\dagger}\right]=\left[a_{2}, a_{2}^{\dagger}\right]=I} \\
& {\left[a_{1}, a_{2}\right]=\left[a_{1}, a_{2}^{\dagger}\right]=\left[a_{1}^{\dagger}, a_{2}\right]=\left[a_{1}^{\dagger}, a_{2}^{\dagger}\right]=0 .}
\end{aligned}
$$

From (10) and (11), it is trivial to show that in this case the Casimir operator becomes

$$
K^{2} \rightarrow-\frac{1}{4}+\frac{1}{4}\left(a_{1}^{\dagger} a_{1}-a_{2}^{\dagger} a_{2}\right)^{2} .
$$

We now introduce the joint number operator eigenstates $|m, n\rangle$,

$$
\begin{aligned}
& |m, n\rangle=(m ! n !)^{-1 / 2}\left(a_{1}^{\dagger}\right)^{m}\left(a_{2}^{\dagger}\right)^{n}|0,0\rangle \\
& a_{1}^{\dagger} a_{1}|m, n\rangle=m|m, n\rangle \quad a_{2}^{\dagger} a_{2}|m, n\rangle=n|m, n\rangle
\end{aligned}
$$

where the notation of separating the eigenvalue labels by a comma should be contrasted with the semicolon convention employed in the previous states defined by (3), so that no confusion can arise. Again, the states $|m, n\rangle$ clearly form an orthonormal basis that spans the entire Hilbert space $H_{2}=H_{1} \otimes H_{1}$ of the two oscillators. In the case where the difference $m-n \equiv \pm n_{0}\left(n_{0}>0\right)$ is a constant integer, we readily see from (12) that $K^{2}=k(k-1) I$, with the (positive) solution $k=\frac{1}{2}\left(1+n_{0}\right)$. As the constant $n_{0}$ runs over the non-negative integers, the index $k$ then runs over values corresponding to the entire series of representations that we have earlier defined as the so-called discrete series. It is also now clear that in the two notations of (3) and (13), the equivalent states

$$
\left|n \pm n_{0}, n\right\rangle \equiv\left|\frac{1}{2}\left(1+n_{0}\right) ; \frac{1}{2}\left(1+n_{0}\right)+n\right\rangle
$$

constitute a basis for the discrete series irreducible unitary representations of the group $\operatorname{SU}(1,1)$.

We now also introduce the usual standard (or atomic or Glauber) joint coherent states $\left|A_{1}, A_{2}\right\rangle$ in $H_{2}$ as simply the product $\left|A_{1}\right\rangle\left|A_{2}\right\rangle$ of each of the standard coherent states for each space $H_{1}$ separately:

$$
\begin{aligned}
& \left|A_{1}, A_{2}\right\rangle=\left|A_{1}\right\rangle\left|A_{2}\right\rangle \equiv U_{1}\left(A_{1}, A_{2}\right)|0,0\rangle \\
& U_{1}\left(A_{1}, A_{2}\right)=U_{1}^{(1)}\left(A_{1}\right) U_{1}^{(2)}\left(A_{2}\right) \\
& U_{1}^{(1)}\left(A_{1}\right) \equiv \exp \left(A_{1} a_{1}^{+}-A_{1}^{*} a_{1}\right) \quad U_{1}^{(2)}\left(A_{2}\right) \equiv \exp \left(A_{2} a_{2}^{+}-A_{2}^{*} a_{2}\right) .
\end{aligned}
$$

As usual, these standard coherent states are readily shown from (15) to be eigenstates of each boson destruction operator,

$$
a_{1}\left|A_{1}, A_{2}\right\rangle=A_{1}\left|A_{1}, A_{2}\right\rangle \quad a_{2}\left|A_{1}, A_{2}\right\rangle=A_{2}\left|A_{1}, A_{2}\right\rangle
$$


In terms of the states $|m, n\rangle$ of definite boson number from (13), one can readily show from (15) that these standard coherent states can be expressed in the equivalent form:

$$
\left|A_{1}, A_{2}\right\rangle=\exp \left[-\frac{1}{2}\left(\left|A_{1}\right|^{2}+\left|A_{2}\right|^{2}\right)\right] \sum_{m=0}^{\infty} \sum_{n=0}^{\infty}(m ! n !)^{-1 / 2} A_{1}^{m} A_{2}^{n}|m, n\rangle .
$$

We note also that these standard coherent states are eigenstates of the $\mathrm{SU}(1,1)$ operator $K_{-} \rightarrow a_{1} a_{2}$,

$$
K_{-}\left|A_{1}, A_{2}\right\rangle=A_{1} A_{2}\left|A_{1}, A_{2}\right\rangle \text {. }
$$

The overlap integral between two such standard coherent states is given by

$$
\begin{aligned}
& \left\langle A_{1}, A_{2} \mid B_{1}, B_{2}\right\rangle=\exp \left[A_{1}^{*} B_{1}+A_{2}^{*} B_{2}-\frac{1}{2}\left(\left|A_{1}\right|^{2}+\left|A_{2}\right|^{2}+\left|B_{1}\right|^{2}+\left|B_{2}\right|^{2}\right)\right] \\
& \left|\left\langle A_{1}, A_{2} \mid B_{1}, B_{2}\right\rangle\right|^{2}=\exp \left(-\left|A_{1}-B_{1}\right|^{2}-\left|A_{2}-B_{2}\right|^{2}\right)
\end{aligned}
$$

and the identity operator in $\mathrm{H}_{2}$ can also be resolved in terms of them as

$$
\pi^{-2} \int \mathrm{d}^{2} A_{1} \mathrm{~d}^{2} A_{2}\left|A_{1}, A_{2}\right\rangle\left\langle A_{1}, A_{2}\right|=I
$$

where $\mathrm{d}^{2} A \equiv \mathrm{d}(\operatorname{Re} A) \mathrm{d}(\operatorname{Im} A)$.

By analogy with the unitary operator $U_{2}$ of (5) that we introduced in I in connection with the representation of $(6)$ of the $\mathrm{SU}(1,1)$ algebra, we now similarly consider an operator $V_{2}$ in connection with the present discrete series representation of $(10)$ :

$$
\begin{aligned}
& V_{2}(\rho, \theta, \lambda) \equiv \exp \left(-\frac{1}{2} \rho \mathrm{e}^{-\mathrm{i} \theta} a_{1}^{\dagger} a_{2}^{\dagger}+\frac{1}{2} \rho \mathrm{e}^{\mathrm{i} \theta} a_{1} a_{2}\right) \exp \left[\mathrm{i} \lambda\left(a_{1}^{\dagger} a_{1}+a_{2}^{\dagger} a_{2}+1\right)\right] \\
& \rho, \theta, \lambda \in \mathbb{R} \quad V_{2}^{\dagger} V_{2}=I=V_{2} V_{2}^{\dagger} .
\end{aligned}
$$

It is straightforward to prove that the two boson creation and destruction operators are unitarily transformed by $V_{2}$ as follows:

$$
\begin{aligned}
& V_{2} a_{1} V_{2}^{\dagger}=\mathrm{e}^{-\mathrm{i} \lambda}\left[\cosh \left(\frac{1}{2} \rho\right) a_{1}+\mathrm{e}^{-\mathrm{i} \theta} \sinh \left(\frac{1}{2} \rho\right) a_{2}^{\dagger}\right] \equiv b_{1} \\
& V_{2} a_{1}^{\dagger} V_{2}^{\dagger}=\mathrm{e}^{\mathrm{i} \lambda}\left[\mathrm{e}^{\mathrm{i} \theta} \sinh \left(\frac{1}{2} \rho\right) a_{2}+\cosh \left(\frac{1}{2} \rho\right) a_{1}^{\dagger}\right]=b_{1}^{\dagger} \\
& V_{2} a_{2} V_{2}^{\dagger}=\mathrm{e}^{-\mathrm{i} \lambda}\left[\cosh \left(\frac{1}{2} \rho\right) a_{2}+\mathrm{e}^{-\mathrm{i} \theta} \sinh \left(\frac{1}{2} \rho\right) a_{1}^{\dagger}\right] \equiv b_{2} \\
& V_{2} a_{2}^{\dagger} V_{2}^{\dagger}=\mathrm{e}^{\mathrm{i} \lambda}\left[\mathrm{e}^{\mathrm{i} \theta} \sinh \left(\frac{1}{2} \rho\right) a_{1}+\cosh \left(\frac{1}{2} \rho\right) a_{2}^{\dagger}\right]=b_{2}^{\dagger} .
\end{aligned}
$$

The inverse transformations are then trivially obtained as

$$
\begin{aligned}
& a_{1}=\mathrm{e}^{\mathrm{i} \lambda} \cosh \left(\frac{1}{2} \rho\right) b_{1}-\exp [-\mathrm{i}(\theta+\lambda)] \sinh \left(\frac{1}{2} \rho\right) b_{2}^{\dagger} \\
& a_{1}^{+}=-\exp [\mathrm{i}(\theta+\lambda)] \sinh \left(\frac{1}{2} \rho\right) b_{2}+\mathrm{e}^{-\mathrm{i} \lambda} \cosh \left(\frac{1}{2} \rho\right) b_{1}^{\dagger} \\
& a_{2}=\mathrm{e}^{\mathrm{i} \lambda} \cosh \left(\frac{1}{2} \rho\right) b_{2}-\exp [-\mathrm{i}(\theta+\lambda)] \sinh \left(\frac{1}{2} \rho\right) b_{1}^{\dagger} \\
& a_{2}^{\dagger}=-\exp [\mathrm{i}(\theta+\lambda)] \sinh \left(\frac{1}{2} \rho\right) b_{1}+\mathrm{e}^{-\mathrm{i} \lambda} \cosh \left(\frac{1}{2} \rho\right) b_{2}^{\dagger} .
\end{aligned}
$$

In view of the unitarity of $V_{2}$ it is clear that the transformation is a canonical one in the sense that the transformed operators obey the same canonical boson commutation relations as their original untransformed counterparts:

$$
\begin{aligned}
& {\left[b_{1}, b_{1}^{\dot{\dagger}}\right]=\left[b_{2}, b_{2}^{\dagger}\right]=I} \\
& {\left[b_{1}, b_{2}\right]=\left[b_{1}, b_{2}^{\dagger}\right]=\left[b_{1}^{\dagger}, b_{2}\right]=\left[b^{\dagger}, b_{2}^{\dagger}\right]=0 .}
\end{aligned}
$$


We also note by direct use of (23) that the operator involved in the Casimir operator of (12) is, as expected, form-invariant:

$$
a_{1}^{\dagger} a_{1}-a_{2}^{\dagger} a_{2}=b_{1}^{\dagger} b_{1}-b_{2}^{\dagger} b_{2} \text {. }
$$

We refer to the transformations of the basic boson operators in (23) as the generalised Bogoliubov transformation. Use of (23), together with the unitarity of $V_{2}$, then further shows how an arbitrary function $f\left(a_{1}, a_{1}^{\dagger}, a_{2}, a_{2}^{*}\right)$ of the basic boson operators transforms under $V_{2}$ as

$V_{2} f\left(a_{1}, a_{1}^{\dagger}, a_{2}, a_{2}^{\dagger}\right) V_{2}^{\dagger}=f\left(b_{1}, b_{1}^{\dagger}, b_{2}, b_{2}^{\dagger}\right) \leftrightarrow V_{2} f\left(a_{1}, a_{1}^{\dagger}, a_{2}, a_{2}^{\dagger}\right)=f\left(b_{1}, b_{1}^{\dagger}, b_{2}, b_{2}^{\dagger}\right) V_{2}$.

The operator $V_{2}$ defines a unitary isomorphism of the Hilbert space $H_{2}$ onto itself. To each state $|s\rangle \in H_{2}$ corresponds the mapped state $V_{2}|s\rangle$, and to each operator $\Theta=$ $\Theta\left(a_{1}, a_{1}^{\dagger}, a_{2}, a_{2}^{\dagger}\right)$ corresponds the mapped operator $\Theta_{\rho, \theta, \lambda} \equiv V_{2} \Theta\left(a_{1}, a_{1}^{\dagger}, a_{2}, a_{2}^{\dagger}\right) V_{2}^{\dagger}=$ $\Theta\left(b_{1}, b_{1}^{\dagger}, b_{2}, b_{2}^{\dagger}\right)$. It is trivial to prove that, if $|s\rangle$ is an eigenstate of $\Theta$, then $V_{2}|s\rangle$ is an eigenstate of $\Theta_{\rho, \theta, \lambda}$ corresponding to the same eigenvalue. We now finally apply these general ideas to the case where the state $|s\rangle$ is one of the standard coherent states $\left|A_{1}, A_{2}\right\rangle$ in $H_{2}$, which we introduced previously in (15).

In this way we are led to introduce the states

$$
\left|A_{1}, A_{2} ; \rho \theta \lambda\right\rangle \equiv V_{2}(\rho, \theta, \lambda)\left|A_{1}, A_{2}\right\rangle=V_{2}(\rho, \theta, \lambda) U_{1}\left(A_{1}, A_{2}\right)|0,0\rangle .
$$

Using (27) we easily prove that they are eigenstates of the mapped destruction operators $b_{1}$ and $b_{2}$ :

$$
\begin{aligned}
& b_{1}\left|A_{1}, A_{2} ; \rho \theta \lambda\right\rangle=A_{1}\left|A_{1}, A_{2} ; \rho \theta \lambda\right\rangle \\
& b_{2}\left|A_{1}, A_{2} ; \rho \theta \lambda\right\rangle=A_{2}\left|A_{1}, A_{2} ; \rho \theta \lambda\right\rangle .
\end{aligned}
$$

The mapped vacuum state $|0,0 ; \rho \theta \lambda\rangle=V_{2}(\rho, \theta, \lambda)|0,0\rangle$ is of particular importance as the vacuum for $b_{1}$ - and $b_{2}$-type bosons, since it obeys the relations

$$
b_{1}|0,0 ; \rho \theta \lambda\rangle=0=b_{2}|0,0 ; \rho \theta \lambda\rangle \text {. }
$$

More generally, (29) shows that the mapped state $\left|A_{1}, A_{2} ; \rho \theta \lambda\right\rangle$ is just a standard coherent state with respect to both b-type bosons, although it is considerably more complex in terms of the original a-type bosons. Thus, in terms of the transformed boson operators, it can be written as

$$
\begin{aligned}
\left|A_{1}, A_{2} ; \rho \theta \lambda\right\rangle & =V_{2}(\rho, \theta, \lambda) U_{1}\left(A_{1}, A_{2}\right)|0,0\rangle \\
& =\exp \left(A_{1} b_{1}^{\dagger}-A_{1}^{*} b_{1}+A_{2} b_{2}^{\dagger}-A_{2}^{*} b_{2}\right) V_{2}(\rho, \theta, \lambda)|0,0\rangle \\
& =\exp \left(A_{1} b_{1}^{\dagger}-A_{1}^{*} b_{1}+A_{2} b_{2}^{\dagger}-A_{2}^{*} b_{2}\right)|0,0 ; \rho \theta \lambda\rangle .
\end{aligned}
$$

Alternatively, it can be written in terms of the states $|m, n ; \rho \theta \lambda\rangle$ of definite particle number for both b-type bosons:

$$
\begin{aligned}
& |m, n ; \rho \theta \lambda\rangle=V_{2}(\rho, \theta, \lambda)|m, n\rangle \\
& b_{1}^{\dagger} b_{1}|m, n ; \rho \theta \lambda\rangle=m|m, n ; \rho \theta \lambda\rangle \\
& b_{2}^{\dagger} b_{2}|m, n ; \rho \theta \lambda\rangle=n|m, n ; \rho \theta \lambda\rangle
\end{aligned}
$$

by analogy with (17), in the form

$\left|A_{1}, A_{2} ; \rho \theta \lambda\right\rangle$

$$
=\exp \left[-\frac{1}{2}\left(\left|A_{1}\right|^{2}+\left|A_{2}\right|^{2}\right)\right] \sum_{m=0}^{\infty} \sum_{n=0}^{\infty}(m ! n !)^{-1 / 2} A_{1}^{m} A_{2}^{n}|m, n ; \rho \theta \lambda\rangle .
$$




\section{Application: the damped quantum oscillator}

As a physical application of the above discrete series of representations of the group SU $(1,1)$, we now turn our attention to the damped quantum harmonic oscillator. This has been previously studied both by the Hamiltonian methods (Feshbach and Tikochinsky 1977, Ghosh and Hasse 1981), which are of interest to us here, and by other means (Kostin 1972, 1975, Immele et al 1975, Svin'in 1975, Stocker and Albrecht 1979). The applicability of two bosonic operators to this problem can be envisaged from the outset, once one recalls that the damped oscillator is characterised by two separate frequencies which describe the (undamped) oscillations and the damping rate.

We start our discussion by consider the classical equations of motion

$$
\begin{aligned}
& \ddot{x}+(R / m) \dot{x}+\omega^{2} x=0 \\
& \ddot{y}-(R / m) \dot{y}+\omega^{2} y=0
\end{aligned}
$$

for, respectively, the damped oscillator (coordinate $x$ ) and its dual (or time-reversed) amplified oscillator (coordinate $y$ ), where the positive constants $m$ and $R$ are respectively the inertial (mass) and damping parameters, and $\omega$ is the natural frequency in the absence of damping or amplification. The classical solutions are

$$
\begin{array}{ll}
x \sim \exp [( \pm \mathrm{i} \varepsilon-R / 2 m) t] & y \sim \exp [( \pm \mathrm{i} \varepsilon+R / 2 m) t] \\
\varepsilon & \equiv\left(\omega^{2}-R^{2} / 4 m^{2}\right)^{1 / 2} .
\end{array}
$$

We assume here and henceforth that $R<2 m \omega$, so that $\varepsilon$ is real, which corresponds to the classical case of under-critical damping. In the opposite case $(R>2 m \omega)$ of over-critical damping there is no classical oscillatory motion superimposed on the damping or amplification. It is readily checked that the Lagrangian

$$
L=m \dot{x} \dot{y}+\frac{1}{2} R(x \dot{y}-\dot{x} y)-m \omega^{2} x y
$$

leads via the usual Euler-Lagrange equations to the equations of motion (34). In turn this Lagrangian leads to the canonically conjugate momenta $p_{x}$ and $p_{y}$, and hence to the Hamiltonian $H$, given by

$$
\begin{aligned}
& p_{x}=m \dot{y}-\frac{1}{2} R y \quad p_{y}=m \dot{x}+\frac{1}{2} R x \\
& H=(1 / m) p_{x} p_{y}+(R / 2 m)\left(y p_{y}-x p_{x}\right)+m \varepsilon^{2} x y .
\end{aligned}
$$

This system may now be quantised by following the standard canonical prescription of replacing the classical $c$-number coordinates $x, y$ and their canonically conjugate momenta $p_{x}, p_{y}$ by operators which satisfy the usual canonical commutation relations:

$$
\begin{aligned}
& {\left[x, p_{x}\right]=\left[y, p_{y}\right]=\mathrm{i} \hbar I} \\
& {[x, y]=\left[x, p_{y}\right]=\left[y, p_{x}\right]=\left[p_{x}, p_{y}\right]=0 .}
\end{aligned}
$$

Alternatively, if one introduces the following linear combinations:

$$
\begin{aligned}
& a_{1} \equiv \frac{1}{2}(m \hbar \varepsilon)^{-1 / 2}\left[\left(p_{x}+p_{y}\right)-\mathrm{i} m \varepsilon(x+y)\right] \\
& a_{1}^{\dagger}=\frac{1}{2}(m \hbar \varepsilon)^{-1 / 2}\left[\left(p_{x}+p_{y}\right)+\mathrm{i} m \varepsilon(x+y)\right] \\
& a_{2} \equiv \frac{1}{2}(m \hbar \varepsilon)^{-1 / 2}\left[\left(p_{x}-p_{y}\right)-\mathrm{i} m \varepsilon(x-y)\right] \\
& a_{2}^{+}=\frac{1}{2}(m \hbar \varepsilon)^{-1 / 2}\left[\left(p_{x}-p_{y}\right)+\mathrm{i} m \varepsilon(x-y)\right]
\end{aligned}
$$


it is easy to show that, if the coordinates and momenta obey the commutation relations (38), then these new operators obey the commutation relations (11) appropriate to two independent bosons. It is easy to show that the Hamiltonian of (37) may be rewritten in terms of the boson operators of (39) in the form

$$
\begin{aligned}
& H \equiv H\{a\}=H_{0}+H_{1} \\
& H_{0}=\hbar \varepsilon\left(a_{1}^{\dagger} a_{1}-a_{2}^{\dagger} a_{2}\right) \quad H_{1}=\frac{i \hbar R}{2 m}\left(a_{1}^{\dagger} a_{2}^{+}-a_{1} a_{2}\right) .
\end{aligned}
$$

We note also the readily proved fact that the two pieces $H_{0}$ and $H_{1}$ of the Hamiltonian written in this form commute:

$$
\left[H_{0}, H_{1}\right]=0 \text {. }
$$

This is important for later purposes.

Before proceeding we note that the presence of damping (and amplification) has necessitated within this formalism the introduction of a second boson, and the consequent 'doubling of the energy levels'. This phenomenon of splitting of the energy levels is by no means restricted to the present example, but rather is a familiar feature in many quantum mechanical situations. We point out that, in the case of zero damping $(R=0)$, the present formalism does not simply reduce to the one-boson case. While the coordinates $x$ and $y$ then obey the same undamped equation of motion, the doubling still remains. One finds normal coordinates $(x \pm y)$, with positive energy for one mode and negative energy for the other mode.

In order to construct the eigenstates of $H$, the commutation relation (41) can be used to find simultaneous eigenfunctions of $H_{0}$ and $H_{1}$, thereby lifting the degeneracy inherent in $H_{0}$ alone. Of particular use in this regard is the general relation

$$
\begin{aligned}
\exp \left(-\gamma K_{+}-\right. & \left.\gamma^{*} K_{-}\right) K_{0} \exp \left(\gamma K_{+}+\gamma^{*} K_{-}\right) \\
& =\cos (2|\gamma|) K_{0}+(2|\gamma|)^{-1} \sin (2|\gamma|)^{-1}\left(\gamma K_{+}-\gamma^{*} K_{-}\right)
\end{aligned}
$$

where $\gamma$ is an arbitrary complex constant, and which is easy to prove, using the commutation relations (1). On putting $\gamma= \pm \pi / 4$, and using the representation of (10), we then find

$$
H_{1} \exp \left[ \pm \frac{1}{4} \pi\left(a_{1}^{\dagger} a_{2}^{\dagger}+a_{1} a_{2}\right)\right]=\mp \frac{i \hbar R}{2 m} \exp \left[ \pm \frac{1}{4} \pi\left(a_{1}^{\dagger} a_{2}^{+}+a_{1} a_{2}\right)\right]\left(a_{1}^{\dagger} a_{1}+a_{2}^{\dagger} a_{2}+1\right) \text {. }
$$

Finally, by making use of the fact that $H_{0}$ commutes with the operator $\left(a_{1}^{\dagger} a_{2}^{+}+a_{1} a_{2}\right)$, we can readily show that eigenstates $\left|\Psi_{n_{1} n_{2}}^{( \pm\}}\right\rangle$of $H$ with corresponding eigenvalues $E_{n_{1} n_{2}}^{( \pm)}$

$$
H\left|\Psi_{n_{1} n_{2}}^{( \pm)}\right\rangle=E_{n_{1} n_{2}}^{( \pm)}\left|\Psi_{n_{1} n_{2}}^{( \pm)}\right\rangle
$$

are given by

$$
\begin{aligned}
& \left.\left|\Psi_{n_{1} n_{2}}^{( \pm t)}=\exp \left[ \pm \frac{1}{4} \pi\left(a_{1}^{\dagger} a_{2}^{\dagger}+a_{1} a_{2}\right)\right]\right| n_{1}, n_{2}\right\rangle \\
& E_{n_{1} n_{2}}^{( \pm)}=\hbar \varepsilon\left(n_{1}-n_{2}\right) \mp \frac{i \hbar R}{2 m}\left(n_{1}+n_{2}+1\right)
\end{aligned}
$$

where the states $\left|n_{1}, n_{2}\right\rangle$ are defined in (13).

At first sight it seems clearly incorrect that the energy eigenvalues of (45) are complex since the Hamiltonian $H$ of $(40)$ undoubtedly appears to be Hermitian. This seeming paradox is resolved upon realising, however, that the normalisation integral for the eigenstates of (45) is divergent, so that $H$ is indeed not a Hermitian operator, 
and the relevant theorem about the reality of its eigenvalues fails to apply. On the other hand, the derivation of the Hamiltonian that we have already given via the classical damped and amplified oscillators certainly leads us to anticipate the complex conjugate pairs of eigenvalues as in (45). However, one big problem remains with the corresponding eigenstates of (45), namely that since they are not normalisable we may not work in the usual Hilbert space. Thus, we are unable to expand an arbitrary state in terms of the eigenstates $\left|\Psi_{n_{1} n_{2}}^{( \pm)}\right\rangle$using the usual metric of the Hilbert space. Feshbach and Tikochinsky (1977) have suggested a possible way to surmount this problem by extending the usual metric of the Hilbert space to include such operators as the Hamiltonian $H$. They do this by defining a new metric wherein the inner product is calculated not with state vectors $\psi_{1}$ and $\psi_{2}^{*}$ as usual but with $\psi_{1}$ and $\psi_{2}^{\mathrm{T}}$, where $\psi_{2}^{\mathrm{T}}$ is the time-reversed version of $\psi_{2}$. In particular we note that $\left|\Psi_{n_{1} n_{2}}^{(+)}\right\rangle$and $\left|\Psi_{n_{1} n_{2}}^{(-)}\right\rangle$are time reverses of each other, and form a biorthogonal set with this new metric.

This important topic has also been discussed by Ghosh and Hasse (1981). While it is not perhaps of immediate relevance for present purposes, we take this opportunity to pursue their discussion at this point in the light of further possible applications of our coherent states. There is no doubt that the method of dual coordinates as employed here does have problems with normalisation, as pointed out by Ghosh and Hasse. This has led some authors to believe that the Lagrangian of Feshbach and Tikochinsky is not a correct formulation for describing the damping of a quantum oscillator. One may take the view that what is actually being described by the formalism is a fictitious Lagrangian system with two degrees of freedom, whose equations of motion are those of a damped oscillator and the corresponding anti-damped oscillator. It seems quite clear that the Lagrangian of (36) does indeed not describe an isolated damped oscillator, but rather a damped oscillator coupled with its amplified counterpart.

Our own viewpoint is that this latter case is just the physically interesting oneparticularly in applications to many-body or field-theoretic systems. We view the situation in complete analogy with the usual interpretation of the way that resonant and anti-resonant pair states arise in many-body systems, where the other (ignored) degrees of freedom are themselves responsible for the creation of the resonance phenomena. It appears to us that it is just because the formalism does not describe an isolated damped quantum oscillator, but rather the dual damped and anti-damped modes, that it may have particular relevance to real fluctuation-dissipation phenomena. We return to this discussion in our concluding remarks in $\S 4$.

We now wish to make use of our transformation $V_{2}$ of (22) to study more general Hamiltonians that can be reached by unitarily transforming the above $H=H\{a\}$ of (40). Equation (27) immediately gives

$$
H^{\prime}\{a\} \equiv V_{2} H\{a\} V_{2}^{\dagger}=H\{b\}=\hbar \varepsilon\left(b_{1}^{\dagger} b_{1}-b_{2}^{\dagger} b_{2}\right)+\frac{i \hbar R}{2 m}\left(b_{1}^{\dagger} b_{2}^{\dagger}-b_{1} b_{2}\right) .
$$

Explicit substitution from (23) then yields the expression

$$
H^{\prime}\{a\}=H^{\prime}=\hbar(\Omega+\varepsilon) a_{1}^{\dagger} a_{1}+\hbar(\Omega-\varepsilon) a_{2}^{\dagger} a_{2}+\hbar \Omega+\kappa a_{1}^{\dagger} a_{2}^{\dagger}+\kappa^{*} a_{1} a_{2}
$$

where

$$
\begin{aligned}
\Omega & \equiv-\frac{R}{2 m} \sin (2 \lambda+\theta) \sinh \rho \\
\kappa & \equiv \frac{\mathrm{i} R \hbar}{2 m} \mathrm{e}^{-\mathrm{i} \theta}[\cos (2 \lambda+\theta)+\mathrm{i} \sin (2 \lambda+\theta) \cosh \rho] .
\end{aligned}
$$


We note that (48) can be inverted to find real $\rho, \theta, \lambda$ for given arbitrary complex $\kappa$ and real $\Omega$, only under the implied restriction that $|\kappa|^{2}-\hbar^{2} \Omega^{2} \equiv(R h / 2 m)^{2}>0$. Conversely, Hamiltonians of the form of (47) can be written in the equivalent form of (46) only for $|\kappa|^{2}>\hbar^{2} \Omega^{2}$. From our general discussion of $\S 2$, we then immediately have that the eigenfunctions and corresponding eigenvalues of the Hamiltonian $H^{\prime}$ of (47), in the case $|\kappa|^{2}>\hbar^{2} \Omega^{2}$, are

$$
\begin{aligned}
\left|\Psi_{n_{1} n_{2}}^{\prime( \pm)}\right\rangle & =V_{2}(\rho, \theta, \lambda) \exp \left[ \pm \frac{1}{4} \pi\left(a_{1}^{+} a_{2}^{+}+a_{1} a_{2}\right)\right]\left|n_{1}, n_{2}\right\rangle \\
& =\exp \left[ \pm \frac{1}{4} \pi\left(b_{1}^{+} b_{2}^{+}+b_{1} b_{2}\right)\right]\left|n_{1}, n_{2} ; \rho \theta \lambda\right\rangle \\
E_{n_{1} n_{2}}^{\prime( \pm)} & =\hbar \varepsilon\left(n_{1}-n_{2}\right) \mp \mathrm{i}\left(|\kappa|^{2}-\hbar^{2} \Omega^{2}\right)^{1 / 2}\left(n_{1}+n_{2}+1\right)
\end{aligned}
$$

with $V_{2}(\rho, \theta, \lambda)$ given by (22) and the $b$ operators given by (23), with parameters $\rho, \theta, \lambda$ found by inverting (48), and with use made of the states defined in (32).

For completeness, we also discuss the case $|\kappa|^{2} \leqslant \hbar^{2} \Omega^{2}$. In this case, the Hamiltonian of (47) cannot be unitarily transformed into that of (46). However, it is of considerable interest to note that even in this case an operator $V_{2}$ of the form of (22) may again be used, now to diagonalise the Hamiltonian. The most general transformed Hamiltonian that can thus be reached from $H^{\prime}$ of (47), for arbitrary $\Omega$ and $\kappa$, may be obtained by substituting the inverse generalised Bogoliubov transformation from (24). In this way we find

$$
H^{\prime}\{a\} \equiv h\{b\}=V_{2} h\{a\} V_{2}^{\dagger}
$$

where we have again used (27), and where

$$
\begin{aligned}
& h\{b\}=\hbar \varepsilon\left(b_{1}^{\dagger} b_{1}-b_{2}^{\dagger} b_{2}\right)+M\left(b_{1}^{\dagger} b_{1}+b_{2}^{\dagger} b_{2}+1\right)+N b_{1}^{\dagger} b_{2}^{\dagger}+N^{*} b_{1} b_{2} \\
& M \equiv \hbar \Omega \cosh \rho-\frac{1}{2}\left(\kappa \mathrm{e}^{\mathrm{i} \theta}+\kappa^{*} \mathrm{e}^{-\mathrm{i} \theta}\right) \sinh \rho \\
& N \equiv \exp [-\mathrm{i}(2 \lambda+\theta)]\left[\kappa \mathrm{e}^{\mathrm{i} \theta} \cosh ^{2}\left(\frac{1}{2} \rho\right)+\kappa^{*} \mathrm{e}^{-\mathrm{i} \theta} \sinh ^{2}\left(\frac{1}{2} \rho\right)-\hbar \Omega \sinh \rho\right] .
\end{aligned}
$$

It is not difficult to see that, by a suitable choice of $\rho$ and $\theta$, either $M$ or $N$ (but not both) may be made zero, depending on whether $\hbar^{2} \Omega^{2}\left\langle|\kappa|^{2}\right.$ or $\hbar^{2} \Omega^{2}>|\kappa|^{2}$ respectively. In the former case, the general Hamiltonian of (47) may be transformed to the Hamiltonian $h \rightarrow H$ of (40) by choosing the parameters $\rho, \theta$ and $\lambda$ to satisfy (48). Conversely, when $\hbar^{2} \Omega^{2}>|\kappa|^{2}$, we can reduce the general Hamiltonian of (47) to the Hamiltonian $h\{b\} \rightarrow \tilde{H}\{b\}$, where

$$
\tilde{H}\{b\}=\hbar \varepsilon\left(b_{1}^{\dagger} b_{1}-b_{2}^{\dagger} b_{2}\right)+\operatorname{sgn} \Omega\left(\hbar^{2} \Omega^{2}-|\kappa|^{2}\right)^{1 / 2}\left(b_{1}^{\dagger} b_{1}+b_{2}^{\dagger} b_{2}+1\right)
$$

by the choice of parameters

$$
\rho=\tanh ^{-1}\left(\frac{|\kappa|}{\hbar \Omega}\right) \quad \theta=-\arg \kappa
$$

and $\lambda$ is arbitrary. In this case we then have that the eigenfunctions and corresponding eigenvalues of the Hamiltonian $H^{\prime}$ of $(47)$, when $\hbar^{2} \Omega^{2}>|\kappa|^{2}$, are given by

$$
\begin{aligned}
& \left|\Psi_{n_{1} n_{2}}^{\prime}\right\rangle=\exp \left[\frac{1}{2|\kappa|} \tanh ^{-1}\left(\frac{|\kappa|}{\hbar \Omega}\right)\left(\kappa^{*} a_{1} a_{2}-\kappa a_{1}^{\dagger} a_{2}^{\dagger}\right)\right]\left|n_{1}, n_{2}\right\rangle \\
& E_{n_{1} n_{2}}^{\prime}=\hbar \varepsilon\left(n_{1}-n_{2}\right)+\operatorname{sgn} \Omega\left(\hbar^{2} \Omega^{2}-|\kappa|^{2}\right)^{1 / 2}\left(n_{1}+n_{2}+1\right) .
\end{aligned}
$$

It is perhaps finally worth pointing out that it seems to be widely believed that an arbitrary Hamiltonian of the form

$$
H^{\prime}=\omega_{1} a_{1}^{\dagger} a_{1}+\omega_{2} a_{2}^{\dagger} a_{2}+\kappa a_{1}^{\dagger} a_{2}^{\dagger}+\kappa^{*} a_{1} a_{2}
$$


can always be diagonalised by a Bogoliubov transformation of the form considered here. On the contrary, what we have shown here is that while the Bogoliubov transformation is always useful, it only reduces the Hamiltonian (55) to diagonal form (i.e. two-mode free form) in the case where the parameter $\Delta \equiv \frac{1}{4}\left(\omega_{1}+\omega_{2}\right)^{2}-|\kappa|^{2}$ is positive. Conversely, when $\Delta$ is negative the Bogoliubov transformation, while still applicable, reduces the Hamiltonian (55) to that of the single damped oscillator given by (40). We note that in both cases the parameter $\Delta$ is left invariant by the transformation.

\section{Conclusions}

We pointed out in I that whereas other authors (Barut and Giradello 1971, Perelomov 1977) have also considered coherent states of the group $\mathrm{SU}(1,1)$, both the states considered by us earlier in I and in the present work are certainly different from those discussed previously by others. We also pointed out that, whereas earlier work hinted at the relationship between these generalised coherent states and the Bogoliubov transformation, our own work aimed to demonstrate this very clearly. Indeed this facet has again been given a central role here. Once more we have seen how starting with the concept of pairing (now between the unlike bosons) leads inexorably via the introduction of the only possible pairing operators to describe the phenomenon, to the Bogoliubov transformation itself. In this context we reiterate that, since we hope as one of our aims to extend this work to provide a more general formalism of all clustering (i.e. higher order than pairing) phenomena, this central feature is of particular relevance. We have again shown that, just as the standard coherent states in the two-boson Hilbert space $H_{2}=H_{1} \otimes H_{1}$ may be defined as simultaneous eigenstates of the original single-boson destruction operators $a_{1}$ and $a_{2}$, so our generalised coherent states, which now provide a representation of the so-called discrete series, may be viewed as simultaneous eigenstates of a pair of new single-boson destruction operators $b_{1}$ and $b_{2}$. These latter operators are themselves generated from the original $a$ operators and their adjoints by the generalised Bogoliubov transformation. In other words our generalised coherent states may either be viewed in terms of pairing between unlike doublets of the original bosons, or as standard coherent states in terms of the unitarily transformed Hilbert space spanned by arbitrary numbers of the two types of Bogoliubov quasiparticles which again obey the usual bosonic canonical commutation relations.

As is by now very well known, standard (Glauber) coherent states have found applicability in a variety of physical situations, typically involving correlation and coherence properties of the underlying quantum fields. One of the reasons for this is clearly that field expansions in terms of the (overcomplete sets of) coherent states greatly facilitate the taking of the appropriate classical limits. This feature is in turn connected with the property of the standard coherent states that they minimise the appropriate Heisenberg uncertainty relation. In fields such as quantum optics or quantum electronics it is usually this property of coherent states that is stressed from the outset, or indeed that is used to define them. Since we have already seen how our generalised paired coherent states may be viewed in terms of the Bogoliubov quasiparticles as standard coherent states, it is to be expected that they will also be of at least theoretical relevance in these fields. Thus in I we remarked how our previous generalised $\mathrm{SU}(1,1)$ paired coherent states, defined via the generators $U_{2}(\rho, \theta, \lambda)$ of (5), could be used in connection with the possibility of a two-photon laser. Similar states have indeed been so introduced into quantum optics (Yuen 1976, Walls 1983), 
where they have become known as squeezed states for reasons discussed earlier in 1 . In the present context, we were particularly interested to learn that very recently Caves and Schumaker (1985) have discussed, in the field of quantum electronics, similar states to the ones defined via the generators $V_{2}(\rho, \theta, \lambda)$ of $(22)$ that we have considered in the present work. However, their connection with the discrete series of representations of SU $(1,1)$ was not discussed by these authors. One of our present hopes is that our own work might extend the range of applicability of these states by stressing some of their broader features based on their underlying group-theoretical foundations.

Indeed, we have been at pains to stress that the unitary operators $V_{2}$ of (22) do not only map the standard coherent states $\left|A_{1}, A_{2}\right\rangle$ in the original Hilbert space $H_{2}=H_{1} \otimes H_{1}$ of the two fundamental types of bosons, into the new paired coherent states $\left|A_{1}, A_{2} ; \rho \theta \lambda\right\rangle$ of (28). Rather, each of the operators $V_{2}$-associated with the entire discrete series of representations of the group $\mathrm{SU}(1,1)$ of original relevance to the problem of pairing-provides a unitary isomorphism of the Hilbert space $\mathrm{H}_{2}$ onto itself. Thus, an arbitrary state $|s\rangle$ of the space is mapped into another state $|s ; \rho \theta \lambda\rangle$ belonging to the space. In particular an arbitrary eigenstate of any operator is mapped by $V_{2}$ into a corresponding eigenstate of the unitarily transformed operator, with the same eigenvalue. We especially made use of this result in our illustration in $\S 3$ of a particular example of the use of this mapping, namely the application to the theory of damped linear harmonic oscillators.

This example is of particular importance as a first step toward a general theory of 'quantum friction'. A quantum mechanical description of dissipation is of importance in many different areas of physical interest. Within quantum optics, for example, the quantum theory of lasers and of photon detection provide examples. On the other hand, there are also several areas in the theory of the atomic nucleus where dissipative processes play a key role. In this respect one may mention both fission and the deep inelastic scattering of heavy ions off nuclei. In the former case, much experimental data indicate that in fission much of the energy associated with the fission degrees of freedom (typically described by a small number of collective coordinates through some such model as the liquid drop model) is transferred, or dissipated, into other channels (typically associated with one- or few-particle degrees of freedom). Similarly, in heavy-ion reactions, it is often observed that a phenomenon known as deep inelastic scattering occurs. In this case a large part of the incident kinetic energy is converted, or dissipated, into internal energy of the reaction products. Such dissipative processes occur very widely in many-body or field-theoretic systems. In the present paper we show in particular how a very wide class of Hamiltonians can be related by our methods to that of the damped linear harmonic oscillator.

We have already alluded in $\$ 3$ to problems associated with normalisation of the states in the Feshbach-Tikochinsky formalism. We view the damped and amplified oscillators described by the model Lagrangian in the same way that we view the resonance and anti-resonance states in many-body systems-namely as very useful approximations to some collective behaviour of the system. One knows that no such resonant or anti-resonant state can be an exact solution to the many-body Schrödinger equation, since the associated energy eigenvalues are not real. However, the dual appearance of the resonant and anti-resonant pairs still enables the total energy to remain real and conserved. The damped and amplified oscillators of the FeshbachTikochinsky (effective) Lagrangian may be viewed in a similar fashion. One knows that the familiar resonant and anti-resonant states (of, for example, atomic physics or condensed matter physics) have problems associated with the fact that they are not 
true energy eigenstates, and hence not proper quantum mechanical states in the many-body Hilbert space. Their infinite norm is a well known example, and we have already mentioned the analogous feature of the Feshbach-Tikochinsky formalism, as discussed by Ghosh and Hasse. In the present case we take the stance that, while the objections raised by Ghosh and Hasse are formally correct, the states may still profitably be viewed as approximations to important collective modes, and their lack of normalisability does not prevent one extracting physical meaning from them. As a basis for this viewpoint we cite the entire corpus of theory of resonant states in many-body systems. It is this viewpoint that also leads us to believe that the present coherent states could have applications to general fluctuation-dissipation phenomena.

Of central importance to us here is that we are able to study canonically the phenomenon of the splitting of the energy levels which arises from the damping process in this model. In particular we contrast our fully canonical description with related semiclassical work. We believe that our method may be very profitably extended to other models where there is a classical degeneracy lifted by quantum tunnelling. Such phenomena, which are again very widespread in quantum field and many-body theory applications, have more usually been treated in the past with various semiclassical approximations.

Finally, it is our hope to extend the present approach beyond the phenomenon of pairing investigated here to include higher clustering correlations. In this way we hope to generalise the associated Bogoliubov transformation to include transformations and the associated generalised coherent states appropriate to clustering phenomena involv. ing more than two particles at a time, and to the condensed matter phases that would presumably arise because of them.

\section{Acknowledgment}

We gratefully acknowledge support for this work in the form of a research grant from the Science and Engineering Research Council of Great Britain.

\section{References}

Bargmann V 1947 Ann. Math., Lpz. 48568

Barut A O and Fronsdal C 1965 Proc. R. Soc. A 287532

Barut A O and Girardello L 1971 Commun. Math. Phys. 2141

Bishop R F and Vourdas A 1986 J. Phys. A: Math. Gen. 192525

Caves C M and Schumaker B L. 1985 Phys. Rev. A 31 3068, 3093

Coleman S 1979 The Whys of Subnuclear Physics (Proc. Int. School of Subnuclear Physics, Erice, 1977) ed A Zichichi (New York: Plenum) p 805

Feshbach H and Tikochinsky Y 1977 Trans. NY Acad. Sci. 3844

Ghosh G and Hasse R W 1981 Phys. Rev. A 241621

Holman W J and Biedenharn L C 1966 Ann. Phys., NY 391

Immele J D, Kan K-K and Griffin J J 1975 Nucl. Phys. A 24147

Kostin M D 1972 J. Chem. Phys. 573589

1975 J. Stat. Phys. 12145

Lax M 1966a Proc. Brandeis Univ. Summer School 1966 vol 2, ed M Chretien, E P Gross and S Deser (New York: Gordon and Breach)

- 1966 b Phys. Rev. 145110

Mollow B R and Glauber R J 1967 Phys. Rev. 1601097

Perelomov A M 1977 Usp. Fiz. Nauk 12323 (Sov. Phys.-Usp. 20 703) 
Senitzky I R 1960 Phys. Rev. 119670

1961 Phys. Rev. 124642

Stocker W and Albrecht K 1979 Ann. Phys., NY 117436

Svin'in I R 1975 Teor. Mat. Fiz. 2292 (Theor. Math. Phys. (USSR) 22 67)

Walls D F 1983 Nature 306141

Yuen H P 1976 Phys. Rev. A 132226 